On voit que la fermentation kéfirique est pratiquement arrêtée au $20^{\circ}$ passage; aussi, B. Boणrounoff conclut-il qu'il s'agit là d'une méthode de préparation défectueuse, et que e'est au procédé russe qu'il faut donner la préférence.

\title{
REVUE
}

\section{LA PASTEURISATION DU LAIT PAR LE COURANT ÉLECTRIQUE}

\author{
par G. GÉNIN
}

Une des principales difficultés que l'on rencontre lorsqu'on procède à la pasteurisation du lait est due à des surchauffes locales qui se produisent dans certains points des appareils et qui communiquent au lait un gô̂t défectueux en diminuant par suite sa qualité. On a donc cherché à réaliser un chauffage du lait, c'est-à-dire une pasteurisation, au moyen du courant électrique, en faisant traverser le lait par ce courant. Les résultats ont été très satisfaisants et actuellement ce procédé, qui aux Etats-Unis est déjà appliqué dans de nombreuses installations, tend à s'étendre de plus en plus en Europe.

Il est possible de ehauffer le lait par courant électrique par suite de la présence dans ce lait de sels minéraux ou d'autres électrolytes en concentration suffisante. Il suffit de faire passer un courant alternatif dans le lait, l'amenée du courant se faisant par des électrodes de carbone et le lait constituant lui-même le milieu chauffant, ce qui évite toute surchauffe plus ou moins localisée.

Depuis que cette technique a été proposée à l'industrie laitière, des améliorations nombreuses ont été apportées à ce procédé, en particulier au cours de ces dernières années. Dans la pratique, une installation de pasteurisation par le courant électrique comprend les appareils suivants :

10 Un réservoir d'alimentation muni d'un flotteur qui règle une vanne et sert à régulariser la pression du liquide pénétrant dans l'appareil proprement dit. De ce réservoir, le lait est conduit dans des réchauffeurs-échangeurs où il est élevé à la température de $49^{\circ}$, la chaleur nécessaire à cette élévation de température étant fournie par le lait chaud sortant du pasteurisateur. A la sortie des réchauffeurs, le lait est pompé au moyen d'une pompe rotative dans un filtre, puis de là dans la chambre de pasteurisation. Cette chambre est constituée par une cuve rectangulaire portant des plaques de carbone sur ses côtés opposés. Ces plaques de carbone constituant les électrodes sont refroidies extérieurement par un courant d'eau froide et elles 
sont alimentées au moyen d'un courant alternatif de 60 périodes sous une tension de 220 volts.

La résistance offerte au passage du courant par le lait entraîne une élévation de la température de ce dernier qui passe de 49 à $72^{\circ}$ ou plus; cette température se trouve d'ailleurs rigoureusement contrôlée et est enregistrée au moyen d'appareils enregistreurs. La durée de passage du lait dans la cuve de pasteurisation est d'environ 16 secondes. A sa sortie le lait chaud passe dans les réchauffeurs où il se refroidit déjà en partie, puis de là dans une série de tubes traversés par un courant de saumure où sa température s'abaisse à environ $4^{\circ} 5$.

Un point que nous n'avons pas signalé plus haut et qui pourtant présente une certaine importance est que le lait traverse l'échangeur de température ou réchauffeur, sous pression réduite. Par suite, si une fuite quelconque se produisait dans l'appareil, le lait déjà pasteurisé reviendrait en arrière et se mélangerait au lait brut et l'inverse ne pourrait pas se produire. A aucun moment, il ne peut done y avoir passage direct du lait brut dans le lait pasteurisé. Le contrôle automatique dont nous avons parlé plus haut consiste tout simplement à faire varier la vitesse du moteur commandant la pompe d'alimentation du filtre. Si la température s'élève trop rapidement dans l'appareil à pasteuriser, on augmente la vitesse de passage du courant de lait et inversement, mais l'intensité du courant électrique reste constante pendant toute la durée de l'opération. Lors de la mise en route de l'installation, on commence par faire passer dans l'appareil de l'èau salée et lorsque le dispositif de contrôle fonctionne régulièrement et que la température ne varie pas de plus de deux degrés, on alimente l'appareil en lait. Tout l'appareillage est en aluminium, sauf les tuyauteries à l'intérieur desquelles circule la saumure réfrigérante.

Les principaux avantages de ce procédé qui a été décrit par C. G. KING dans une communication faite au soixante-deuxième congrès de la Société d'Electrochimie américaine, à qui nous avons d'ailleurs emprunté ces renseignements, sont dus à ce que le chauffage est effectué avec une uniformité remarquable, le lait étant exposé le moins possible, soit à l'air sóit aux métaux. Chaque partie du lait se trouve uniformément chauffée, le degré de chauffage dépendant en effet de la résistance du lait qui est uniforme si le produit est homogène. Avec un tel procédé, on évite totalement les surfaces chauffantes et il s'ensuit que les surchauffes locales sont supprimées, en même temps que le non chauffage et par suite la non pasteurisation des mousses. Enfin l'absence d'oxydation atmosphérique et l'absence d'un contact prolongé du lait dans les métaux permet de supprimer l'action de ces derniers qui en tant que cata- 
lyseurs oxydants avaient comme inconvénient de réduire l'activité en vitamine $\mathrm{C}$ naturelle du lait.

En même temps que l'installation d'un appareil de pasteurisation électrique du lait permet de réduire sensiblement l'encombrement à débit égal, une telle installation qui fonctionne d'une façon continue et dont le contrôle est automatique n'exige qu'une très faible dépense de main-d'œuvre, ce qui équilibre et très largement l'augmentation de dépense provenant du remplacement du chauffage à la vapeur par le chauffage électrique.

En dehors des avantages d'ordre technique que présente la pasteurisation électrique du lait, ce nouveau mode de traitement de cet aliment essentiel possède également des avantages d'ordre purement chimique et bactériologique et nous reproduirons dans ce qui suit quelques résultats obtenus par des bactériologistes allemands et hollandais qui montrent l'épuration remarquable qu'assure la pasteurisation électrique.

Nous indiquerons tout d'abord des résultats de laboratoire obtenus en traitant le lait à une tension d'environ 2.600 volts et avec une intensité de 1 ampère. Lorsque, traité dans ces conditions, le lait a atteint une température de $66^{\circ}$, il est pratiquement exempt de colibacilles et le tableau ci-dessous reproduit d'après 100 expériences le pourcentage moyen de microbes par rapport au pourcentage initial d'un lait chauffé électriquement à des températures variables :

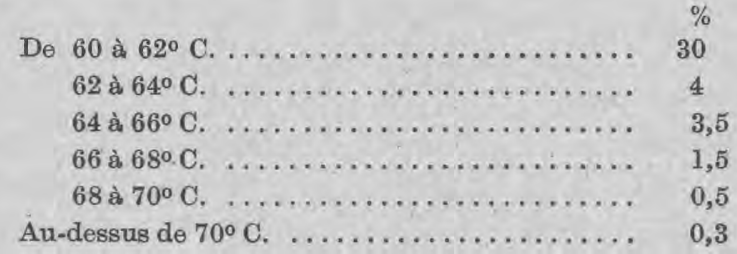

C'est à la suite de ces résultats très encourageants qu'un appareil construit d'après le brevet Aten-Lulofs fut examiné après avoir fonctionné dans quelques grandes laiteries d'Amsterdam par le Service municipal pour l'inspection des denrées alimentaires d'Amsterdam et par la station de contrôle du lait de la même ville (Dr Plimster et Dr von Gelder).

Dans le tableau ci-dessous, nous avons reproduit les résultats obtenus par ces deux derniers savants sur des laits prélevés à différents endroits de l'appareil de pasteurisation électrique :

Traitement du lait

Nombre moyen de microbes au centimètre cube

Température $80.85^{\circ}$, lait recueilli immédiatement à la sortie de

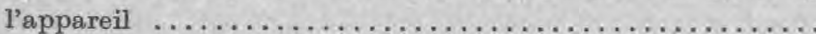


Température $80-85^{\circ}$, lait recueilli avant la mise en bouteille, après passage dans l'appareil, l'échangeur-récupérateur et le réfrigérant $\ldots \ldots \ldots \ldots \ldots \ldots \ldots \ldots \ldots \ldots \ldots \ldots \ldots \ldots \ldots \ldots \ldots \ldots \ldots$

Température $80-85^{\circ}$, maintenue à $80^{\circ}$ pendant 5 minutes, lait recueilli après passage dans l'échangeur-récupérateur avant la mise en bouteille $\ldots \ldots \ldots \ldots \ldots \ldots \ldots \ldots \ldots \ldots \ldots$

Température $80-85^{\circ}$, maintenue à $80^{\circ}$ pendant 10 minutes, lait recueilli après passage dans l'échangeur-récupérateur et le réfrigérant $\ldots \ldots \ldots \ldots \ldots \ldots \ldots \ldots \ldots \ldots \ldots \ldots \ldots \ldots \ldots \ldots$

Température $80-85^{\circ}$, maintenue à $80^{\circ}$ pendant 20 minutes, lait recueilli après passage dans l'échangeur-réeupérateur et le réfrigérant $\ldots \ldots \ldots \ldots \ldots \ldots \ldots \ldots \ldots \ldots \ldots \ldots \ldots \ldots \ldots \ldots \ldots \ldots$

Dans tous les cas, les échantillons examinés étaient exempts de colibacilles et donnaient une réaction de Storch négative. On pouvait donc considérer le lait comme parfaitement pasteurisé, puisqu'il ne contenait même pas le dixième du nombre de bactéries admissibles dans un lait pasteurisé.

Il est intéressant de souligner que le nombre de bactéries survivantes ne diminue pas sensiblement lorsque le lait après son passage normal dans l'appareil est maintenu même pendant 20 minutes à la température de pasteurisation. Comme nous l'avons dit plus haut, le lait qui traverse la totalité des différentes parties de l'appareil en 48 secondes ne reste dans le pasteurisateur proprement dit que pendant 16 secondes et, pendant ce temps très court, la pasteurisation est tellement efficace qu'elle n'est nullement améliorée lorsque l'on prolonge la durée de chauffage.

Dans le tableau suivant, on a publié les résultats d'essais effectués sur le lait immédiatement recueilli dans des récipients stérilisés et par conséquent non susceptible de se polluer. Ces résultats font ressortir mieux encore l'excellente qualité du lait obtenu :

Traitement du lait

Nombre de mierobes au centimètre cube agar gélatine

Lait frais innombrable $\quad 36.800$

Température $84-85^{\circ}$, lait pris immédiatement à la sortie de l'appareil . . . . . . . . . . . . . . . .

Température $84-85^{\circ}$, lait ayant passé dans l'échangeur-récupérateur et le réfrigérant $\ldots \ldots \ldots \ldots \ldots$

Température $84-85^{\circ}$, maintenue à $80^{\circ}$ pendant 7 minutes $\ldots \ldots \ldots \ldots \ldots \ldots \ldots \ldots \ldots \ldots$

Température $84-85^{\circ}$, maintenue à $80^{\circ}$ pendant 15 minutes $\ldots \ldots \ldots \ldots \ldots \ldots \ldots \ldots \ldots$

$600 \quad$ stérile

400 200

stérile 800

stérile 
Ces résultats démontrent également que le lait pris à la sortie de l'appareil et ayant traversé cet appareil à la vitesse normale est aussi bien pasteurisé que le lait qui est resté soumis pendant 15 minutes à la température de pasteurisation.

En ce qui concerne le goût du lait, l'expérience a montré que le lait recueilli immédiatement à la sortie de l'appareil ne se distingue pas du lait frais. Le lait maintenu par chauffage électrique à la température de $80^{\circ} \mathrm{C}$. pendant 20 minutes présente un léger goût de pasteurisation, mais il diffère néanmoins à son avantage du lait pasteurisé en bouteille par les méthodes courantes.

Ainsi qu'il fallait s'y attendre, en raison du petit nombre de bactéries, le lait pasteurisé électriquement se conserve très bien, et au bout de 5 jours il est encore dans un état irréprochable.

Le Laboratoire de la "Preussische Versuchs und Forschungsanstalt für Milchwirtschaft » de Kiel a effectué de son côté un certain nombre d'essais en vue de déterminer les caractères du lait pasteurisé électriquement. Cet examen avait pour but principal de déterminer quelle influence le courant électrique pouvait exercer sur les différents types de bactéries que renferme le lait et sur les propriétés physiologiques ou chimiques de cet aliment. On a constaté en premier lieu qu'au-dessus de $65^{\circ} \mathrm{C}$., le nombre total de bactéries n'était plus que de 0,1 à $1 \%$ du nombre de bactéries initial ; à une température supérieure à $68^{\circ} \mathrm{C}$. tous les colibacilles étaient détruits, on pouvait done considérer qu'un lait pasteurisé à $70^{\circ}$ ne renfermait pratiquement plus de bactéries nuisibles.

En ce qui concerne les bactéries productrices d'acidité, l'expérience a montré que l'on trouvait dans un lait pasteurisé électriquement une proportion très favorable entre les bactéries productrices et les bactéries non productrices d'acidité, puisque $50 \%$ des bactéries qui survivent après une pasteurisation à $70^{\circ} \mathrm{C}$. sont productrices d'acidité. Rappelons à ce sujet qu'avec les autres appareils de pasteurisation et en particulier avec les types de pasteurisation autres, ce pourcentage est sensiblement inférieur.

On a enfin examiné la pasteurisation avec un appareil électrique d'un lait infecté de bacilles de la tuberculose. L'expérience a montré qu'une température d'environ $70^{\circ} \mathrm{C}$. était déjà suffisante pour exterminer ces bacilles. La pasteurisation électrique exerce done une action destructive qui n'avait été jusqu'à présent atteinte par aucun des autres appareils de pasteurisation qui à la température de $70^{\circ} \mathrm{C}$. ne détruisent même pas les microbes courants.

On peut donc déduire de ce qui précède que le courant électrique exerce une action spécifique et que la destruction complète des bactéries pathogènes qui se produit entre 60 et $70^{\circ}$ doit être attribuée non seulement à une action thermique, mais également à une 
action électrique au sujet de Iaquelle nous manquons encore de renseignements précis.

Au début de cette étude, nous avons indiqué que le chauffage électrique du lait, si l'on tenait compte de tous les facteurs intervenant sur le prix de revient de la pasteurisation, n'était pas plus coûteux que le chauffage par la vapeur par exemple. Evidemment il est difficile de donner des chiffres exacts en ce qui concerne ces frais de pasteurisation, car ils dépendent du prix du courant, de la température du lait pénétrant dans l'appareil et de la température de pasteurisation.

On admet qu'en chauffant à une température moyenne de $70^{\circ}$, il faut dépenser environ $85 \mathrm{kw}$. par 1.000 litres de lait si on n'emploie pas d'échangeur-récupérateur et environ $45 \mathrm{kw}$. en utilisant un échangeur-récupérateur.

On peut également, toujours dans un but économique, chauffer préalablement le lait au moyen d'eau chaude à la température de $60^{\circ}$ par exemple et le pasteuriser par le courant électrique qui élève sa température à environ $75^{\circ} \mathrm{C}$. ; cela permet de réaliser une importante économie d'énergie électrique puisqu'il ne faut plus dans ce cas que $18 \mathrm{kw}$. pour 1.000 litres de lait. Si enfin, sans utiliser ce préchauffage par l'eau chaude, on dispose dans l'installation deux échangeurs-récupérateurs, la consommation est d'environ $25 \mathrm{kw}$. par 1.000 litres de lait.

Une laiterie utilisant le système de pasteurisation électrique sera dans tous les cas un client important pour les sociétés de distribution d'énergie et elle pourra exiger de ce fait des tarifs relativement avantageux. D'autre part, en règle générale, la pasteurisation du lait se faisant au début du travail, la consommation d'énergie électrique sera élevée à un moment où les centrales ne fournissent pas une grande quantité de courant; les laiteries pourront done, arguant de ce fait, réclamer des conditions meilleures pour la fourniture de leur courant.

En résumé, la pasteurisation électrique présente sùr les systèmes anciens de pasteurisation des avantages techniques indiseutables. La question des frais de pasteurisation, tout en intervenant dans le choix des usagers, ne doit pas constituer un facteur prépondérant, car une étude approfondie de la question montre qu'il doit être possible par des ententes judicieuses d'obtenir des tarifs avantageux qui rendent l'emploi du courant plus économique. 